\title{
Rethinking C-reactive protein
}

\section{Steve Edelson \& Gaspar Taroncher-Oldenburg, Executive Editors}

An international team has conclusively shown that C-reactive protein does not have a causal role in the chronic inflammatory response at the root of coronary heart disease. ${ }^{1}$ But companies with CRP programs and other researchers think there's still a clear rationale for dampening levels of the protein following acute myocardial infarction or stroke, two conditions that trigger high levels of CRP, which in turn activate the complement system, leading to exacerbated, inflammation-driven tissue damage.

Levels of C-reactive protein (CRP) can increase as part of a systemic, nonspecific response to infection, injury or inflammation. More than two decades ago, elevated levels of the protein were associated with the risk of atherosclerosis, the most common underlying cause of coronary heart disease (CHD), based on the discovery by Mark Pepys and colleagues that aggregates of CRP bind to low-density lipoprotein (LDL) and very low-density lipoprotein (VLDL). ${ }^{2}$

Collaborative studies in which Pepys participated also showed that increased baseline CRP values in the general population are significantly associated with increased risk of CHD but that the association is much weaker than originally reported. The problem, he told SciBX, is that some other researchers "conflated association with causality." Pepys is professor of medicine and director of the Centre for Amyloidosis and Acute Phase Proteins at the University College London Medical School.

"Elevated levels of CRP have by now been clearly associated with risk of cardiovascular disease," said Garret FitzGerald, director of the Institute for Translational Medicine and Therapeutics at the University of Pennsylvania and chair of UPenn's Department of Pharmacology. However, a key outstanding question, he said, has been "whether CRP might actually play a causative role in disease pathogenesis."

In the case of $\mathrm{CHD}$, according to the new results reported in the Journal of the American Medical Association, the answer is a definitive no. The researchers ran a genomewide association study using samples from 17,967 people to identify SNPs related to CRP levels. Although the group found five loci that were significantly associated with CRP levels, subsequent Mendelian randomization studies of the variants in more than 28,000 heart disease patients and 100,000 controls "showed no association with coronary heart disease," according to the authors.

Mendelian randomization essentially strips genetic association data from any potentially confounding factors such as environmental variables. The technique allows researchers to determine whether alleles are associated, independently from other traits, with a specific phenotype such as CHD.

The team was led by Paul Elliott, head of the Department of Epidemiology and Public Health in the faculty of medicine at Imperial College London, and included researchers from over a dozen other institutions worldwide.

The findings were accompanied by a commentary by Svati Shah and James De Lemos, who said the results "make it much less likely that therapies specifically altering CRP levels alone will prove beneficial" in CHD. ${ }^{3}$ Shah is an assistant professor of cardiology at Duke University and De Lemos is an assistant professor of cardiology at The University of Texas Southwestern Medical Center at Dallas.

Shah and De Lemos said the group's data "strongly challenge a causal effect of CRP levels on CHD." They added that "it is likely that CRP does not cause CHD and that reported associations between CRP and CHD are either confounded by other risk factors or represent reverse causality."

FitzGerald agreed. "CRP seems very unlikely to mediate cardiovascular events in humans," he said. "This was a heroic international collaboration using a very nice strategy of Mendelian randomization. Gene variants that substantially influenced levels of CRP did not contribute detectably to cardiovascular risk."

According to Pepys, "the results lay to rest the long-standing controversy" about whether CRP has a causal role in heart disease.

\section{What's the question?}

"These studies can't completely disprove a causal relation, but they may dampen enthusiasm," said Nancy Cook, associate professor of medicine at Harvard Medical School and Brigham and Women's Hospital. "But even if CRP itself is not a causal mediator, it's still a clinically useful marker for inflammation and a strong predictor of cardiovascular disease."

Indeed, whether or not CRP has a causal role in cardiovascular disease is the wrong question to be asking, according to Paul Ridker, director of the Center for Cardiovascular Disease Prevention at Brigham and Women's Hospital and professor of medicine at Harvard Medical School.

Instead, he told SciBX, "the question of causality relates to inflammation," which he and others have argued is likely causal in atherosclerosis.

Indeed, it has been more than 20 years since evidence began to accumulate that cardiovascular disease in general is linked to inflammation and that in particular the process that produces inflammation in the vessel wall is a central player in atherosclerosis and thus CHD. 


\section{TARGETS \& MECHANISMS}

"None of the data presented in the JAMA paper negates this nor reduces the public health importance of the inflammation hypothesis," Ridker said. "Further, even if CRP is only a marker, it remains highly valuable clinically."

There are two biotechs with disclosed programs targeting CRP-Isis Pharmaceuticals Inc. and Pentraxin Therapeutics Ltd. Both told SciBX that modulating CRP should still have clinically relevant effects in cardiovascular indications, even if the target doesn't have a causal role in disease.

"With drugs in general, people get confused and think that we're asking causal questions-we're usually not. What we're asking is: when we reduce CRP levels, do we make people better?" said Stanley Crooke, chairman and CEO of Isis.

He added: "I'm not in the business of finding the causes of disease. I want tools that improve disease."

Crooke noted that the work by Elliott et al. was an epidemiological study. Such studies, he said, "never provide absolutely conclusive results. The answer to every epidemiological study is another epidemiological study. That continues until someone does a hypothesis-testing study where an item such as CRP is directly manipulated-that's the way science draws conclusions."

ISIS 353512, a second-generation antisense molecule that targets CRP, is in Phase I testing to treat multiple indications in which CRP is elevated, such as cardiovascular diseases, rheumatoid arthritis (RA), Crohn's disease and end-stage renal disease (ESRD).

The company hopes to start Phase II testing this year. That program, said Crooke, initially will look at multiple indications including RA and ESRD. After those results are reported, he said the company hopes to look at the compound in acute coronary syndromes (ACSs).

Crooke noted that the JAMA article focused on chronic cardiovascular conditions such as CHD. "I've never been convinced that lowering CRP would be of benefit in chronic outcomes," he said. "In myocardial infarction and acute coronary syndromes, the evidence is much more compelling that lowering CRP should do some good."

Pepys, who also is founder and director of Pentraxin, agreed on this point.

"In atherosclerosis and related diseases, CRP is clearly not involved. In atherothrombosis, the jury is still out on whether CRP is a player or not," he said. "In acute myocardial infarction, however, CRP has definitely been implicated through its active role in triggering complement activation, which in turn causes inflammation. Therefore, efforts to target CRP therapeutically in the latter indication should prove a winning proposition."

Pentraxin is developing the CRP inhibitor bis(phosphocholine)hexane and related compounds for acute myocardial infarction (AMI). The company is seeking funding for Phase I and Phase IIa studies.

Edelson, S. \& Taroncher-Oldenburg, G. SciBX 2(27); doi:10.1038/scibx.2009.1063

Published online July 16, 2009

\section{REFERENCES}

1. Elliott, P. et al. JAMA; published online July 1, 2009; doi:10.1001/jama.2009.954

Contact: Paul Elliott, Imperial College, London, U.K. e-mail: p.elliott@imperial.ac.uk

2. de Beer, F.C. et al. J. Exp. Med. 156, 230-242 (1982)

3. Shah, S. et al. JAMA 301, 92-93 (2009)

\section{COMPANIES AND INSTITUTIONS MENTIONED}

Brigham and Women's Hospital, Boston, Mass.

Duke University, Durham, N.C.

Harvard Medical School, Boston, Mass.

Imperial College London, London, U.K.

Isis Pharmaceuticals Inc. (NASDAQ:ISIS), Carlsbad, Calif.

Pentraxin Therapeutics Ltd., London, U.K.

University College London Medical School, London, U.K. University of Pennsylvania, Philadelphia, $\mathrm{Pa}$.

The University of Texas Southwestern Medical Center at Dallas, Dallas, Texas 